\title{
Kinematic analysis of spiral structures in the local disk
}

\author{
T. Yano, M. Chiba, and N. Gouda \\ National Astronomical Observatory, Mitaka, Tokyo 181-8588, Japan \\ Received 19 July 2001 / Accepted 12 March 2002

\begin{abstract}
We present a method, based on the kinematic analysis of the Galactic disk stars near the Sun, to clarify whether the internal motions of the stellar system in spiral arms follow those expected in the density wave theory. The method is based on the linear relation between the phases of spatial positions and those of epicyclic motions of stars, as predicted by the theory. The application of the method to the 78 Galactic Cepheids near the Sun, for which accurate proper motions are available from the Hipparcos Catalogue, has revealed that these Cepheids show no correlation between both phases, mainly because of the large uncertainties in their spatial motions. This implies that previous studies for the calibration of the wave parameters using a similar sample to ours lack a clear physical basis: the currently available data of the Cepheids are still inaccurate to assess the presence of density waves in the solar neighborhood. We show the required accuracy for the astrometric data of the Cepheids to clarify the issue and future prospects are outlined.
\end{abstract}

Key words. galaxy: kinematics and dynamics - galaxy: disk - galaxy: general - galaxy: structure

\section{Introduction}

Spiral structures of galaxies have been studied for a long time in order to understand how these structures are formed (e.g., Roberts et al. 1975; Rohlfs 1977; Binney \& Tremaine 1987). One of the proposed models to explain spiral arms is that they are just material arms, where the stars originally making up a spiral arm remain in the arm even at a later time. However, this simple model suffers from the well-known "winding problem": the differential rotation in galactic disks winds up the arm in a short time compared with the age of galaxies, so that the spiral pattern would be too tightly wound compared with the observed spiral structures. In contrast, the currently most popular model, which is free of the winding problem, is the density wave theory (Lin \& Shu 1964), where a spiral arm is regarded as a stellar supported wave propagating through galactic disks. In this picture, the global spiral pattern is sustained independently of individual stars moving at different angular velocities. For a comprehensive review of the density wave theory, see, e.g., Rohlfs (1977) and Binney \& Tremaine (1987).

The presence of density waves has been suggested by various lines of observational evidence in spiral galaxies, including the relative distributions of dust lanes, interstellar gas, and H II regions across the arms (Fujimoto 1968; Roberts 1969; Rohlfs 1977), the intensity distribution of radio continuum radiation (Mathewson et al. 1972), and systematic variation of gaseous velocity fields near

Send offprint requests to: T. Yano, e-mail: yano@pluto.mtk.nao.ac.jp the arms (e.g., Visser 1980). In particular, recent highresolution observations using $\mathrm{CO}$ emission have revealed detailed streaming motions of molecular gas, which are generally in agreement with predictions of the density wave models (e.g., Kuno \& Nakai 1997; Aalto et al. 1999). However, we note that these observational results provide only one outcome of the nonlinear interaction between interstellar matter and background stellar arms, and it is yet unknown whether the motions of the stars themselves, which make up the spiral pattern, actually follow those predicted by the density wave theory. In this regard, direct access to detailed stellar kinematics in disks is possible only in our Galaxy.

Here, we present a method to clarify this issue, based on the analysis of the local kinematics of disk stars. We then apply the method to 78 Cepheids in the solar neighborhood, for which the precise data of proper motions are available from the Hipparcos Catalogue (ESA 1997). Also, the distances to these sample stars can be accurately estimated from the period-luminosity relation, so that combined with the radial velocity data, the full threedimensional velocities are available. We note here that although we focus on the local kinematics of spiral arms in this work, the method we develop here can be applied to the motions of more remote stars distributed over a whole disk, for which precise astrometric data will be provided by the next-generation satellites such as FAME and GAIA.

Our paper is organized as follows. In Sect. 2, we describe the method to determine whether or not the motions of stars accord with those expected in the density 


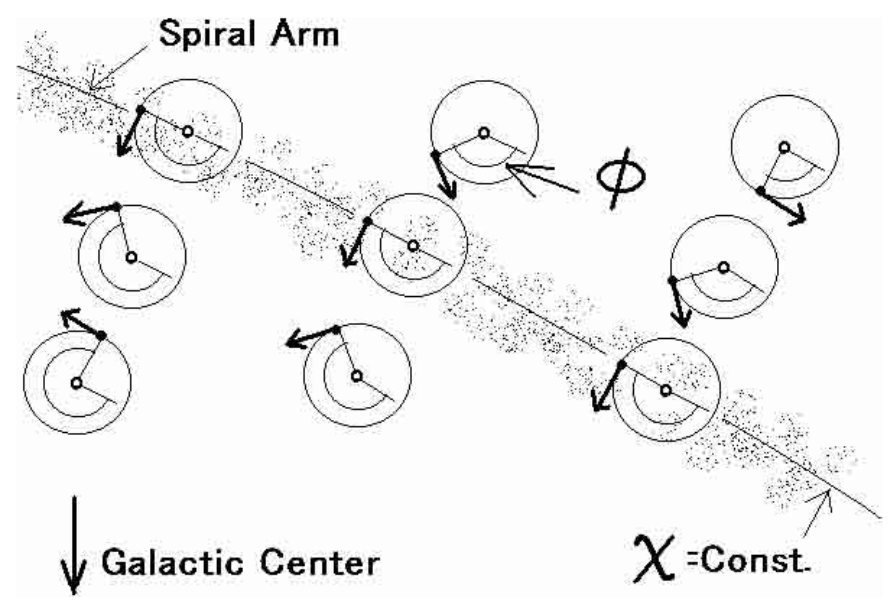

Fig. 1. Schematic diagram showing the definition of phases, $\chi$ and $\phi$, in the Galactic Plane. Each vector corresponds to a velocity vector of each star in epicyclic motion. We note that epicycles are not truly circles, but ellipses, and that the velocity vectors are in the frame of the guiding center.

wave theory. In Sect. 3, we describe the sample stars and the fundamental parameters of our Galaxy adopted in this work. The application of our method to the sample stars is shown in Sect. 4. Finally, Sect. 5 is devoted to discussion and conclusions.

\section{Method}

All orbits of the disk stars in our Galaxy are not perfectly circular. The discrepancy between the motion of a star and its reference circular orbit, called orbit of the guiding center, can be represented by an epicyclic motion of the star (Binney \& Tremaine 1987). We describe here a method to compare the epicyclic motions of the stars observed near the Sun with those expected in the density wave theory.

First, we define the "position phase", $\chi$, as a function of the position of a star in the Galactic plane, by assuming that the shape of the spiral arm is logarithmic as follows:

$\chi=\ln \frac{\left(r / r_{\mathrm{g}}\right)}{\tan i}-\theta+\Omega_{\mathrm{p}} t$

where $r$ is the distance between the star and the Galactic Center (GC), $r_{\mathrm{g}}$ is the distance between the Sun and GC, $i$ is the pitch angle of the spiral arm, $\theta$ is the angle between $r$ and $r_{\mathrm{g}}$, and $\Omega_{\mathrm{p}}$ is the angular velocity of the spiral pattern in the azimuthal direction. We note that all the stars on the same arm have the same value of $\chi$ (Fig. 1).

Second, we define the phase of the epicyclic motion of a star, $\phi$, which is the angle between the direction of a spiral arm and that of a star from the epicenter (Fig. 1) ${ }^{1}$. Then, if the stellar motions in the arm obey the density wave theory, all the stars on the same arm have the same

\footnotetext{
1 We note that the phase $\phi$ deduced from the observed motion of a star contains the observational error (associated with the error in distance and proper motion) and its velocity dispersion as described later. So, Fig. 1 shows an ideal case where only the spiral forcing works.
}

value of $\phi$ as well as the same value of $\chi$. Also, when the number of arms is $m$, the epicyclic frequency $\kappa$ of the stellar motions is equal to $m$ times as large as the angular frequency $\Omega-\Omega_{\mathrm{p}}\left[\kappa=m\left(\Omega-\Omega_{\mathrm{p}}\right)\right]$. If so, since $\mathrm{d} \phi / \mathrm{d} t=-\kappa$, and $\mathrm{d} \chi / \mathrm{d} t=\Omega_{\mathrm{p}}-\mathrm{d} \theta / \mathrm{d} t=\Omega_{\mathrm{p}}-\Omega$ from Eq. (1), we obtain the following linear relation between $\phi$ and $\chi$,

$\phi \equiv m \chi+\phi_{0} \quad(\bmod 2 \pi)$

if the stellar motions obey the density wave theory, where $\phi_{0}$ is a constant. One may postulate that this relation is valid only at the inner Lindblad resonance. However, it is valid even at different radii where spiral arms appear, if the gravitational interaction of a star with the perturbed potential is self-consistently taken into account. Indeed, Contopoulos (1986) shows that closed orbits can be constructed even at radii different from the Lindblad resonance.

Thus, by assessing this linear relation between observed $\phi$ and $\chi$, it is possible to investigate whether or not the observed stellar motions follow those predicted by the density wave theory. We can also derive the number of arms, $m$, from the slope in the $\phi-\chi$ relation. Here we notice that $m$ is positive (negative) at radii inside (outside) the corotation resonance in Eq. (2). In Fig. 3, we show the case $m=4$ (solid lines) as an example.

We also make a distinction between epicyclic motion related to velocity dispersion and epicyclic motion induced by spiral forcing. The latter satisfies the relation (2) and the former behaves as a part of the dispersion in the phase, $\phi$.

In order to obtain the phase, $\phi$, we need the velocity parallel to the arm, $v_{\text {arm,p }}$, and the velocity perpendicular to the arm, $v_{\text {arm,t }}$.

$v_{\mathrm{arm}, \mathrm{t}}=\left(v_{\mathrm{r}}-v_{\mathrm{r}, \text { model }}\right) \cos (\alpha)-\left(v_{\mathrm{t}}-v_{\mathrm{t}, \text { model }}\right) \sin (\alpha)$,
$v_{\mathrm{arm}, \mathrm{p}}=\left(v_{\mathrm{r}}-v_{\mathrm{r}, \text { model }}\right) \sin (\alpha)+\left(v_{\mathrm{t}}-v_{\mathrm{t}, \text { model }}\right) \cos (\alpha)$,

where, $v_{\mathrm{r}}$ and $v_{\mathrm{t}}$ are the radial and the tangential velocities, subtracting the components of the speed of the Sun. Parameters $v_{\mathrm{r} \text {,model }}$ and $v_{\mathrm{t} \text {,model }}$ are the radial and the tangential components of the velocity derived from the axisymmetric potential model. The angle $\alpha$ is the sum of the galactic longitude, $l$, the angle between a star and the Sun from the galactic center, $\theta$, and pitch angle $-i$, i.e., we obtain the following form:

$\alpha \equiv l+\theta-i$

Then we obtain the phase $\phi$ by

$\phi=\arctan \left(\frac{v_{\mathrm{arm}, \mathrm{t}}}{v_{\mathrm{arm}, \mathrm{p}}}\right)$

Here we note that Eq. (6) is exactly valid only if the epicycles induced by spiral forcing are circles. Accordingly, when the epicycles are not circles, this equation is an approximation. 


\section{Data}

We adopt the Galactic Cepheids as tracers of stellar motion in spiral arms, because these bright stars can be seen from a long distance and so we can investigate a wide region around the Sun. Also, these young populations show only a small deviation from circular rotation, thereby allowing us to analyze the internal kinematics of spiral arms alone; relatively old stars, such as dwarf stars, have too large velocity dispersions, possibly due to repeated gravitational interactions with massive clouds (Spitzer \& Schwarzschild 1953) in addition to the effect of spiral arms. Furthermore we can obtain accurate distances for Cepheids, based on the relation between pulsation period and absolute magnitude. We do not use the Hipparcos parallaxes, which have generally large errors for many Cepheids beyond the distance $d \sim 100$ pc from the Sun. We adopt the Cepheid catalog compiled by Mishurov et al. (1997) for distances and radial velocities, and the Hipparcos Catalogue for proper motions, to calculate the individual motions of the Cepheids in the Galactic plane. Here we note that kinematics of the innermost Cepheids may be affected by the Galactic bar.

In the Mishurov et al. catalog, Cepheids in the region of $d>4 \mathrm{kpc}$ and those in binary systems are excluded. Also, nearby Cepheids in the region of $d<0.5 \mathrm{kpc}$ are excluded in order to reduce the local effects, like Gould's Belt. Furthermore, Cepheids whose pulsation periods exceed 9 days are also excluded, because they are supposed to be extremely young objects. In addition, we further exclude the Cepheids within $1 \mathrm{kpc}$ for the following reason. When we compare the observed distribution of Cepheids in the $\phi-\chi$ plane with the theoretical prediction in a quantitative manner, as will be described later, we assign larger statistical weights to the Cepheids having a smaller observational error (Eq. (7)). This leads to larger weights being given to the Cepheids located close to the Sun, say $d<1 \mathrm{kpc}$, so that the result of the analysis will be largely determined by only a small number of Cepheids in a small region near the Sun. We also exclude two Cepheids whose peculiar velocity is exceptionally large, over $50 \mathrm{~km} \mathrm{~s}^{-1}$, compared to other ones. As a consequence, we adopt 78 Cepheids in this work, and their spatial distribution is shown in Fig. 2.

We adopt $r_{\mathrm{g}}=8.3 \mathrm{kpc}$ in our analysis, which is approximately an average of observed values ranging from $8.1 \mathrm{kpc}$ to 8.5 kpc (Kerr \& Lynden-Bell 1986; Hanson 1987; Pont et al. 1994; Feast \& Whitelock 1997). As for the pitch angle of spiral arms, $i$, in our Galaxy, several authors have investigated one of the conspicuous spiral arms near the Sun, the Sagittarius arm, based on the spatial distributions of open clusters, CO emissions, or O-B2 clusters, and have arrived at several to about $20^{\circ}$ (Pavlovskaya \& Suchkov 1984; Dame et al. 1986; Grabelsky et al. 1988; Alfaro et al. 1992; Vallee 1995). In this work, we adopt $i=-12.0^{\circ}$ in the solar neighborhood, where the negative value for $i$ denotes a trailing arm. We have found that even if we change the values of these parameters over a

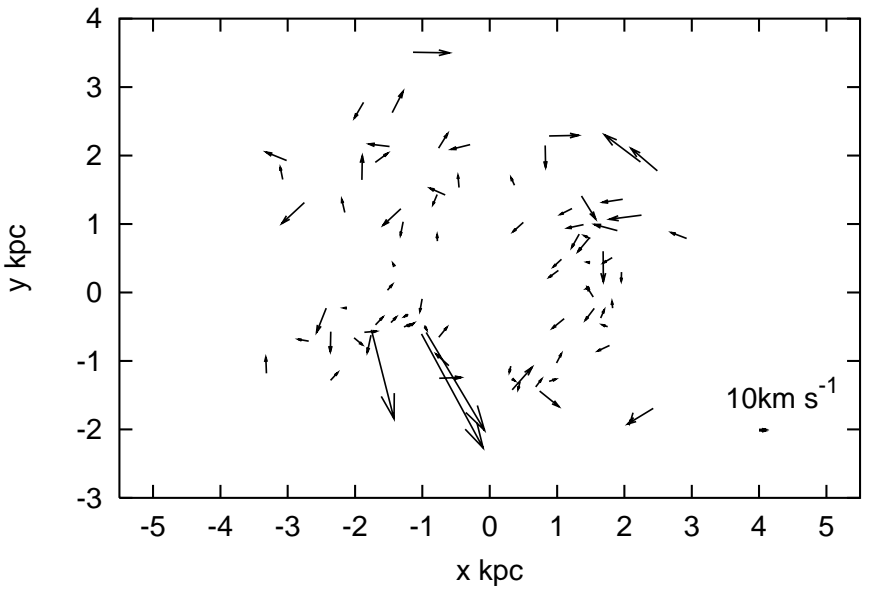

Fig. 2. Positions of the Cepheids in the Galactic plane. The position of the Sun is the origin of the coordinate axes. The Galactic Center is located in the negative direction of the $y$-axis with $x=0$. The plotted arrows indicate residual velocities. A sample of the velocity of $10 \mathrm{~km} \mathrm{~s}^{-1}$ is shown in the lower right corner.

likely range, the result shown below remains essentially unchanged.

In order to analyze the epicyclic motion of the Cepheids, we need to subtract both the local solar motion with respect to the local standard of rest and the effect of the Galactic differential rotation from the observed motions. As the local solar motion, we adopt $15.5 \mathrm{~km} \mathrm{~s}^{-1}$ in the direction of $l_{\odot}=45^{\circ}$ and $b_{\odot}=23.6^{\circ}$ (Kulikovskij 1985). To estimate the effect of the Galactic differential rotation, we assume that in the concerned region within about $4 \mathrm{kpc}$ from the Sun, the angular frequency $\Omega$ of a Galactic star is linearly changing with distance from the Sun, where the Oort constant $A$ is assumed to be $14.5 \mathrm{~km} \mathrm{~s}^{-1} \mathrm{kpc}^{-1}$ (e.g., Kerr \& Lynden-Bell 1986; Feast \& Whitelock 1997). As the local circular velocity, we adopt $220 \mathrm{~km} \mathrm{~s}^{-1}$. We again note that the result shown in Sect. 4 is essentially independent of the value of $A$ or the assumption for differential rotation.

\section{Results}

We present the relation between the phases of the epicyclic motions of the Cepheids, $\phi$, and their position phases, $\chi$, in Fig. 3. We also show the case for the 4-armed galaxy that obeys the density wave theory (solid lines). If the motions of the Cepheids in the solar neighborhood are in accordance with those expected in the density wave theory, we expect a similar linear relation between their $\phi$ and $\chi$. However, such a linear relation is not clearly seen, which may imply either that these objects in the region we investigate do not obey the density wave theory or that the errors in their $\phi$ and $\chi$ are too large.

To be more quantitative, we analyze the motions of the Cepheids by using the sum of squares of the deviations for 


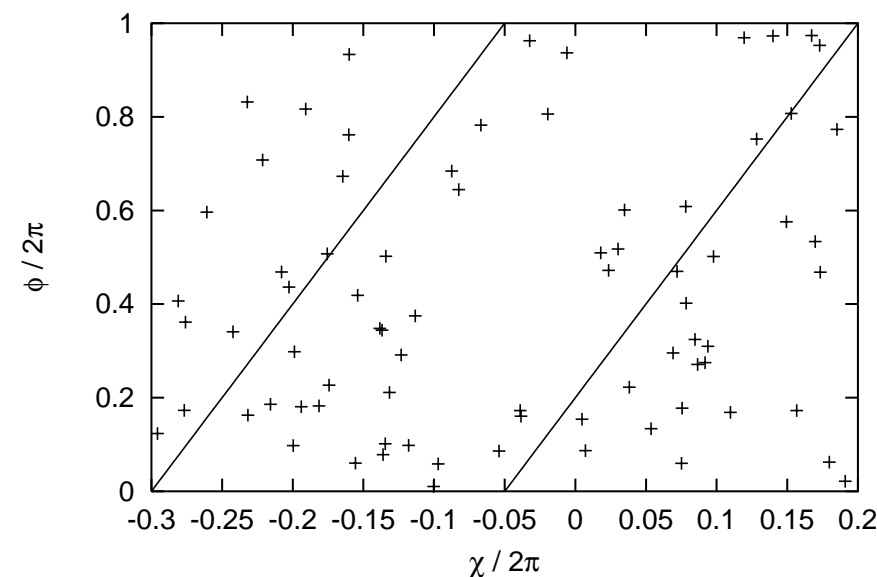

Fig. 3. The phase $\phi / 2 \pi$ plotted against $\chi / 2 \pi$ for our sample of Cepheids. The solid lines show the relation expected for a 4-armed galaxy that obeys the density wave theory.

the observed $\phi$ 's from the theoretically expected ones. We define this deviation, $\Delta^{2}$, as follows:

$\Delta^{2} \equiv \sum_{i} \frac{\delta \phi_{i}^{2}}{\sigma_{i}^{2}}$,

where $\delta \phi_{i}$ is defined as,

$\delta \phi_{i} \equiv \min \left(\left|\phi_{i}-\phi_{\mathrm{DW}}\left(\chi_{i}\right)\right|, 2 \pi-\left|\phi_{i}-\phi_{\mathrm{DW}}\left(\chi_{i}\right)\right|\right)$,

where $\sigma_{i}$ is the error in the phase $\phi_{i}$ of the star $i$, and $\phi_{\mathrm{DW}}\left(\chi_{i}\right)$ is the phase when the motion of the star $i$ obeys the density wave theory.

The error is described by

$$
\begin{aligned}
\delta \phi_{i} & =\frac{\partial \phi_{i}}{\partial v_{\mathrm{r}}} \delta v_{\mathrm{r}}+\frac{\partial \phi_{i}}{\partial v_{\mathrm{t}}} \delta v_{\mathrm{t}} \\
\sigma^{2}=<\delta \phi_{i}^{2}> & =<\left(\frac{\partial \phi_{i}}{\partial v_{\mathrm{r}}}\right)^{2} \delta v_{\mathrm{r}}^{2}+\left(\frac{\partial \phi_{i}}{\partial v_{\mathrm{t}}}\right)^{2} \delta v_{\mathrm{t}}^{2}> \\
& =<\cos ^{2} \psi_{i} \frac{\delta v_{\mathrm{r}}^{2}}{v^{2}}+\sin ^{2} \psi_{i} \frac{\delta v_{\mathrm{t}}^{2}}{v^{2}}>
\end{aligned}
$$

where $v$ is a scalar velocity, $\delta v_{\mathrm{r}}, \delta v_{\mathrm{t}}$ are the errors of the radial and tangential velocities, respectively. The value $\psi_{i}$ is the angle between the line of sight to the star $i$ and the direction of the star from its epicenter. The brackets $<>$ are the ensemble mean of stars. The error $\sigma_{i}$ in the phase $\phi_{i}$ consists of three parts:

$\sigma_{i}^{2}=\sigma_{\text {disp }}^{2}+\sigma_{\text {mag }}^{2}+\sigma_{\mathrm{PM}, i}^{2}$,

where $\sigma_{\text {disp }}$ denotes the error associated with the velocity dispersion of the Cepheids, $\sigma_{\text {mag }}$ the error associated with the uncertainty in their apparent magnitude (typically 0.2 mag which corresponds to $10 \%$ error in the distance), and $\sigma_{\mathrm{PM}, i}$ the error associated with the uncertainty in their Hipparcos proper motion. We adopt $13 \mathrm{~km} \mathrm{~s}^{-1}$ for the velocity dispersion of the Cepheids. These adopted values correspond to $\sigma_{\text {disp }} \simeq 1.7$ and $\sigma_{\text {mag }} \sim 0.01 \ll \sigma_{\text {disp }}$. It is worth noting that to estimate the true error in $\phi_{i}$, we need to know the epicyclic motion of a star alone which is unaffected by the observational error and velocity dispersion.

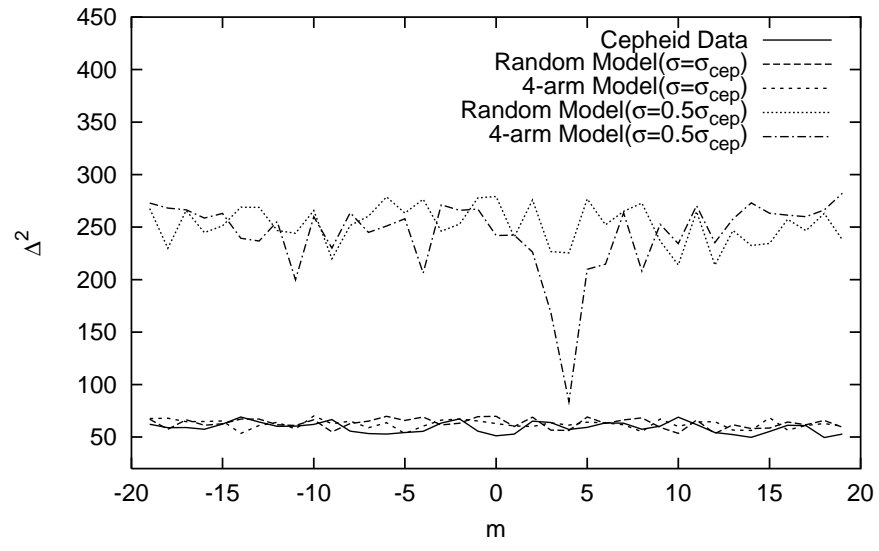

Fig. 4. The sum of square of the deviations, $\Delta^{2}$, as a function of arm-number $m$. Positive $m$ represents the arms at radii inside the corotation resonance, whereas negative $m$ represents those at the outer radii. The solid, dashed, thin dashed, dotted, and dash-dotted lines denote the Cepheid data, random model $(\sigma=$ $\left.\sigma_{\text {cep }}\right)$, 4-arm model $\left(\sigma=\sigma_{\text {cep }}\right)$, random model $\left(\sigma=0.5 \sigma_{\text {cep }}\right)$, and 4-arm model $\left(\sigma=0.5 \sigma_{\text {cep }}\right)$, respectively. Here $\sigma_{\text {cep }}$ stands for the error in the phase $\phi_{i}$, due to the velocity dispersion of the Cepheids and observational error.

However this is not available, as the measured $\phi_{i}$ already includes the error. In this paper, we adopt $10 \mathrm{~km} \mathrm{~s}^{-1}$ as the fiducial velocity from the epicyclic motion. The quantity $\delta \phi_{i}$ denotes the discrepancy between $\phi_{i}$ and $\phi_{\mathrm{DW}}\left(\chi_{i}\right)$, for which we take the smaller value of $\left|\phi_{i}-\phi_{\mathrm{DW}}\left(\chi_{i}\right)\right|$ and $2 \pi-\left|\phi_{i}-\phi_{\mathrm{DW}}\left(\chi_{i}\right)\right|$, because $\phi_{i}$ has a period of $2 \pi$. The phase $\phi_{\mathrm{DW}}\left(\chi_{i}\right)$ is determined by minimizing the deviation $\Delta^{2}$ for each value of the arm number $m$.

If the motions of stars are in accordance with those expected in the density wave theory, the expected value of the deviation $\Delta^{2}$ is about the same as the number of the sample stars (78 in this work), because the distribution of $\delta \phi_{i}^{2}$ is normalized by its standard dispersion $\sigma_{i}^{2}$. Thus, in this case, we will obtain a minimum of $\Delta^{2}$ with $\Delta_{\text {min }}^{2} \lesssim 80$ at a specific arm number $m=m_{\mathrm{g}}$, whereas at other $m$, the value of $\Delta^{2}$ will be systematically larger than 80 ; the larger ratio of $\Delta^{2} / \Delta_{\text {min }}^{2}$ implies that it is more likely that the observed stellar motions match the density wave theory. If $\Delta^{2}$ is always larger than 80 at all $m$, we may conclude that the stellar motions are totally inconsistent with those expected in the theory. If $\Delta^{2}$ is always equal to or smaller than 80 at all $m$, then no statistically meaningful conclusion can be drawn, as there exists no noticeable minimum of $\Delta^{2}$.

We plot the deviation $\Delta^{2}$ as a function of $m$ in Fig. 4 . The solid line shows the result for our Cepheid sample. It is apparent that $\Delta^{2}$ stays always under 80 for all values of the parameter $m$, without showing a noticeable minimum.

In order to examine the statistical significance of this result, we investigate four hypothetical models for comparison. As the first model, we randomly select $\phi$, independently of $\chi$, so that there is no correlation between $\phi$ and $\chi$. We then assign the same errors $\sigma_{i}$ to $\phi_{i}$ as those for the Cepheids, hereafter denoted as $\sigma_{\text {cep }}$. 
The thick dashed line in Fig. 4 shows this case, referred to as "random model". The second model, as shown by the thin dashed line, follows the density wave theory with $m=4$ and the same error $\sigma_{\text {cep }}$ is assigned. As the value of $\Delta^{2}$ also stays less than 80 for all $m$ in this case, it is concluded that there are no significant differences between the properties of these two models and the Cepheids.

We examine two more cases. The third and fourth models are similar to the first model ("random model") and the second model respectively, except for the assignment of smaller errors $\sigma_{i}=0.5 \sigma_{\text {cep }}$, in order to see the effect of reducing $\sigma_{i}$ on the result. In the third model, the value of $\Delta^{2}$ is always larger than 80 at all $m$ without a noticeable minimum. On the other hand, in the fourth model, the value of $\Delta^{2}$ is always around 250 for all $m$, except for the case of $m=4$ at the minimum of $\Delta^{2}\left(\Delta_{\text {min }}^{2} \sim 80\right)$. These experiments suggest us that if the errors are sufficiently small, it is possible to distinguish the third model from the fourth one. In other words, it is possible to assess whether the motions of the stars follow those expected in the density wave theory.

Thus, it is concluded that the large errors in the current Cepheid data prevent us from clarifying the presence of density-wave motions. In this regard, we note that Mishurov et al. (1997) derived the basic parameters of the density waves in our Galaxy, such as their pattern speed, based on a similar sample to ours. Our current experiments however indicate that their work lacks a physical basis.

\section{Discussion and conclusions}

We have presented a method, based on the analysis of local kinematics of disk stars, to clarify whether the motions of the stars in the solar neighborhood follow those expected in the density wave theory. The method utilizes the expected linear relation between the "position phases" of the stars $\chi$ and those of their epicyclic motions $\phi$, as given in Eq. (2). The application of the method to the 78 Galactic Cepheids within $4 \mathrm{kpc}$ from the Sun, for which accurate proper motions are available from Hipparcos, has revealed that an expected linear relation between $\chi$ and $\phi$ is not apparent. Based on the quantitative analysis using the deviation $\Delta^{2}$, we conclude that the currently available data including large observational errors are inaccurate for use in assessing the presence of density-wave motions in the solar neighborhood, so that previous studies for the calibration of the wave parameters using a similar sample to ours (e.g. Mishunov et al. 1997) lack a clear physical basis.

We here investigate to what extent the error in the phase $\phi$ should be reduced so as to extract the possible wave motions of the stars. As shown in Eq. (12), the error $\sigma_{i}$ consists of the error associated with the velocity dispersion of the Cepheids, $\sigma_{\text {disp }}$, the error associated with their apparent magnitude, $\sigma_{\text {mag }}$, and the error in Hipparcos proper motions, $\sigma_{\mathrm{PM}, i}$. The former two effects are basically unavoidable from the analysis, so we focus on the latter effect associated with the uncertainty in

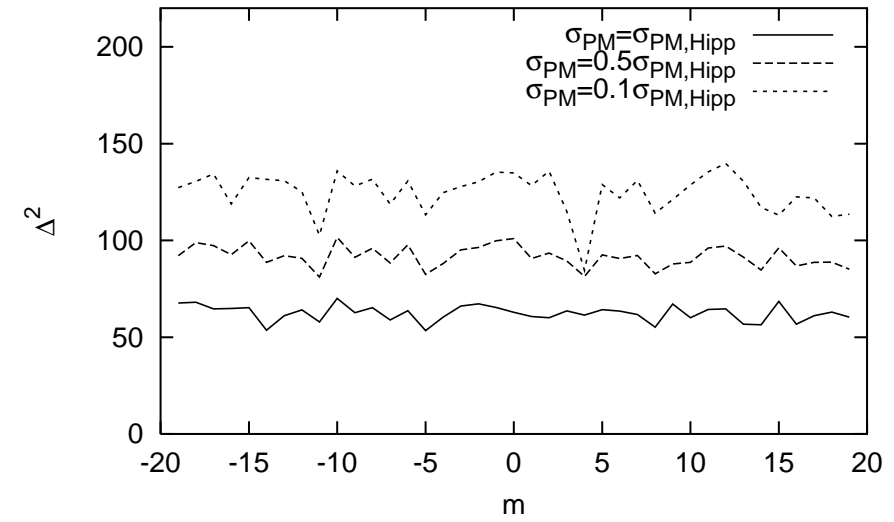

Fig. 5. The relation $\Delta^{2}$ vs. $m$ for the three 4 -arm models with different errors related to proper motions, $\sigma_{\mathrm{PM}}$. The solid, dashed, and thin dashed lines denote the 4-arm models with $\sigma_{\mathrm{PM}}=\sigma_{\mathrm{PM}, \mathrm{Hipp}}$ (i.e. the same as the second model in Fig. 4), $\sigma_{\mathrm{PM}}=0.5 \sigma_{\mathrm{PM}, \mathrm{Hipp}}$, and $\sigma_{\mathrm{PM}}=0.1 \sigma_{\mathrm{PM}, \mathrm{Hipp}}$, respectively. Here $\sigma_{\mathrm{PM}, \mathrm{Hipp}}$ stands for the error associated with the uncertainty of the Hipparcos proper motion for each Cepheid.

the measurement of proper motions. Three representative models following density waves with $m=4$, where different values of $\sigma_{\mathrm{PM}, i}$ are assigned, are examined and the results are shown in Fig. 5 . In the first model, $\sigma_{\mathrm{PM}, i}$ is the same as that for the current Cepheid data using Hipparcos proper motions, referred to as $\sigma_{\mathrm{PM} \text {,Hipp }}$ (i.e. the same as the second model in Fig. 4). In the second and third models, we adopt the smaller errors, $\sigma_{\mathrm{PM}, i}=0.5 \sigma_{\mathrm{PM} \text {,Hipp }}$ and $0.1 \sigma_{\mathrm{PM}, \text { Hipp }}$, respectively. As is evident in Fig. 5 , the third model shows a noticeable minimum of $\Delta^{2}$ at $m=4$, whereas in the first and second models there exists no minimum. Thus, these experiments suggest that if the error in proper motion measurement is less than a tenth of that for Hipparcos proper motion, then it is possible to obtain a minimum in $\Delta^{2}$ and so to extract wave motions of stars. Indeed, the next-generation satellites such as FAME and GAIA will provide very precise proper motions: the error is expected to be about 10 times smaller than that in the Hipparcos Catalogue, thereby enabling us to assess the density wave theory. If we adopt the value of under $10 \mathrm{~km} \mathrm{~s}^{-1}$ as the fiducial velocity from the epicyclic motion, it is difficult to obtain a noticeable minimum.

Even if the motions of the stars generally follow those expected in the density wave theory, there are some other possibilities that may affect the results.

First, the spiral structure around the Sun may not be as simple as that given in Eq. (1). In fact, many of our sample Cepheids belong to the local spiral structure called the Orion arm, for which a definite conclusion on its spatial structure is yet to be reached. It is frequently expressed as the "Orion spur", having a rather irregular pattern compared to other large-scale arms, Sagittarius and Perseus arm (see, e.g., Gilmore et al. 1989). The existence of the Orion arm may cause disturbances in the density wave motion of stars, induced by these large-scale arms.

Second, the Cepheids we have adopted here may still convey the systematic velocities of the dense gas clouds 
from which these stars were formed, in the form of the streaming motions. If there still exist some individual streaming motions among the sample stars, such motions may violate the ideal linear relation between $\chi$ and $\phi$ expected for the density wave motions.

In order to examine the last issue described above more clearly, we have repeated our analysis using younger populations with smaller velocity dispersions than the Cepheids. As such young stars, we have adopted the O-B5 stars, although due to their fainter luminosities than Cepheids, the sample with available proper motions is confined to the narrower region near the Sun. These sample stars are taken from the NASA SKY2000 Master Star Catalog Ver. 2 (Sande et al. 1998) which provides almost 300000 stars brighter than $8 \mathrm{mag}$. The catalog contains many basic quantities, such as MK classification, luminosity class, apparent magnitude, color, radial velocity, and so on. We have then calibrated distances using Hipparcos parallaxes or spectroscopic distances using the program kindly supplied by Drs. M. Sôma and M. Yoshizawa, and also obtained accurate proper motions by the cross-identification with the Hipparcos and ACT Reference Catalogs (Urban et al. 1998). After removing binaries and multiples, we selected 773 O-B5 stars for which full three-dimensional velocities are available. Then, the application of the method we have developed here revealed that the deviation $\Delta^{2}$ as a function of $m$ has qualitatively the same behavior as the case of the Cepheids, i.e., we cannot obtain any noticeable minimum at a specific value of $m$. In this case also, we cannot clarify whether the motions of the stars follow those expected in the density wave theory. It is also the case that even if the motions of the stars accord with with those expected in the density wave theory, the results may be affected by the proximity of the stars: most of these O-B5 stars are located within $\sim 1 \mathrm{kpc}$ from the Sun.

More definite conclusions on the issue we have addressed here require the assembly and analysis of much larger numbers of stars with accurate distances and proper motions, so that the statistical fluctuation in the result can be significantly reduced. Also, it is necessary to assemble the data of more remote stars over a large fraction of the disk, thereby diminishing effects of local irregular spiral structures on the kinematic analysis. Indeed, as emphasized here, the next-generation satellites such as FAME and GAIA will provide very precise astrometric data for huge numbers of Galactic stars and will thus offer us an opportunity to assess detailed motions of disk stars in conjunction with the density wave theory.

Acknowledgements. We thank the referee, Dr. G. Bertin, for his constructive criticism which helped us to improve the paper. We are also grateful to M. Sôma and M. Yoshizawa for providing us with the program for calculating spectroscopic distances. T.Y. would like to thank K. Okoshi and S. Bouquillon for useful discussion. This work was supported in part by Research Fellowships of the Japan Society for the Promotion of Science for Young Scientists (No. 00074).

\section{References}

Aalto, S., Huetemeister, S., Scoville, N. Z., \& Thaddeus, P. 1999, ApJ, 522, 165

Alfaro, E. J., Cabrera-Cano, J., \& Delgado, A. J. 1992, ApJ, 399,576

Binney, J. J., \& Tremaine, S. 1987, Galactic Dynamics (Princeton University Press, Princeton, NJ)

Contopoulos, G., \& Grosbol, P. 1986, A\&A, 155, 11

Dame, T. M., Elmegreen, B. G., Cohen, R. S., \& Thaddeus, P. 1986, ApJ, 305, 892

ESA 1997, The Hipparcos and Tycho Catalogues (ESA SP1200) (Noordwijk: ESA)

Feast, M., \& Whitelock, P. 1997, MNRAS, 291, 683

Fujimoto, M. 1968, in Non-Stable Phenomena in Galaxies, (Yerevan: Armenian Academy of Sciences), IAU Symp., 29 453

Gilmore, G., King, I., \& van der Kruit, P. 1989, The Milky Way as a Galaxy, 19th Advanced Course of the Swiss Society of Astrophysics and Astronomy, ed. R. Buser, \& I. King, SAAS-FEE

Grabelsky, D. A., Cohen, R. S., Bronfman, L., \& Thaddeus, P. 1988, ApJ, 331, 181

Hanson, R. B. 1987, AJ, 93, 816

Kerr, F., \& Lynden-Bell, D. 1986, MNRAS, 221, 1023

Kulikovskij, P. G. 1985, Zvezdnaya Astronomiya (Stellar Astronomy), Nauka, Moscow, USSR (in Russian)

Kuno, N., \& Nakai, N. 1997, PASJ, 49, 279

Lin, C. C., \& Shu, F. H. 1964, ApJ, 140, 646

Mathewson, D. S., van der Kruit, P. C., \& Brouw, W. N. 1972, A\&A, 17, 468

Mishurov, Yu. N., Zenina, I. A., Dambis, A. K., Mel'Nik, A. M., \& Rastorguev, A. S. 1997, A\&A, 323, 775

Pavlovskaya, E. D., \& Suchkov, A. A. 1984, Soviet Astron., 28, 389

Pont, F., Mayor, M., \& Burki, G. 1994, A\&A, 285, 415

Roberts, W. W. 1969, ApJ, 158, 123

Roberts, W. W., Roberts, M. S., \& Shu, F. H. 1975, ApJ, 196, 381

Rohlfs, K. 1977, Lectures on Density Wave Theory, Lecture Notes in Physiscs 69, ed. J. Ehlers, K. Hepp, R. Kippenhahn, H. A. Weidenmuller, \& J. Zittartz (SpringerVerlag)

Sande, C. B., Brasoveanu, D., Miller, A. C., Home, A. T., Tracewell, D. A., \& Warren, W. H. Jr. 1998, Spaceflight Dynamics, 100, Part 1, Advances in Astronautical Sciences, in Proc. of the AAS/GSFC International Symposium on Space Flight Dynamics, ed. T. H. Stengle (American Astronautical Society Publication), 765

Spitzer, L., \& Schwarzschild, M. 1953, ApJ, 118, 106

Urban, S. E., Corbin, T. E., \& Wycoff, G. L. 1998, AJ, 115, 2161

Vallee, J. P. 1995, ApJ, 454, 119

Visser, H. C. D. 1980, A\&A, 88, 159 